\title{
The LuNa project: experimental didactic modules exploiting portable setups to teach optics in primary and secondary schools
}

Maria Bondani, Alessia Allevi, Luca Nardo, Fabrizio Favale

Maria Bondani, Alessia Allevi, Luca Nardo, Fabrizio Favale, "The LuNa project: experimental didactic modules exploiting portable setups to teach optics in primary and secondary schools," Proc. SPIE 9289, 12th Education and Training in Optics and Photonics Conference, 92892D (17 July 2014); doi: 10.1117/12.2070730

Event: 12th Education and Training in Optics and Photonics Conference, 2013, Porto, Portugal 


\title{
The "LuNa" Project: experimental didactic modules exploiting portable setups to teach optics in Primary and Secondary Schools
}

\author{
Maria Bondani ${ }^{a, b, c}$, Alessia Allevi ${ }^{b, c}$, Luca Nardo $^{d}$ and Fabrizio Favale ${ }^{b}$ \\ ${ }^{a}$ Institute for Photonics and Nanotechnologies, CNR, Como, Italy \\ ${ }^{b}$ Dipartimento di Scienza e Alta Tecnologia, Università degli Studi dell'Insubria, Como, Italy \\ ${ }^{c}$ CNISM UdR Como, Como, Italy \\ ${ }^{d}$ Dipartimento di Scienze della Salute - Università degli Studi di Milano Bicocca, Milano, Italy
}

\begin{abstract}
The "LuNa" (La natura della Luce nella luce della Natura - The nature of Light in the light of Nature) Project is devoted to the experimental teaching of optics in the different school grades. The basic idea of the Project is that the history of optics and the debate about the nature of light are a meaningful example of how science proceeds in the development of a physical model. Moreover optical phenomena can be presented at different levels of complexity in order to be accessible to students of different age. At the core of the Project are several portable setups that support experimental and partially interactive lectures covering all the aspects of optical phenomena, from geometrical optics to single-photon interference passing through atmospheric optics, spectroscopy, holography and theory of perception. When possible, the setups are realized with simple and easy to find materials so as to be reproducible by teachers and students. Of course, for the most complicated setups (interferometers and holography) research materials are used. Each module is calibrated to fit teachers' requirements either to be included in the curricula of their classes or to be used as an expansion of the optics program.
\end{abstract}

Keywords: Primary and Secondary Schools, geometric optics, wave optics, portable experimental setups

\section{INTRODUCTION}

In our Country scientific curricula seem to have a reduced capacity to attract students, though they offer skills important to spend on a professional level. One of the reasons for this phenomenon lies in the fact that science subjects are often experienced by students as too difficult and unattractive in content presentations, which almost always happen through frontal lectures. On the other hand, the wonder and curiosity about natural phenomena, and about the discovery of the mechanisms that govern them are features inherent in all human beings, especially in the younger. We have therefore found it necessary to devise new strategies and innovative educational pathways in the transmission of scientific knowledge and skills, exploiting the innate curiosity of students, by starting, for example, from the observation of the natural world and by intensively using laboratory activities. Unfortunately, not all educational institutions have the necessary equipment to realize suitable experimental activities and, above all, teachers are not always accompanied and encouraged to acquire the skills necessary to implement this kind of teaching.

The objective of LuNa Project ${ }^{1}$ is twofold: on the one hand, we want to keep alive the students' natural interest in the reality of natural phenomena, on the other hand, we want to pursue a substantial improvement in the standard level of teaching of science subjects at each school level, by supporting the upgrade of teachers and offering them the opportunity to see how to perform laboratory experiments.

The Project deepens its roots into the experience in didactics and science dissemination gained over the years by the Authors and is designed to optimize, harmonize and make available to the schools a number of laboratory experiments of Physics, designed to fit into the ministerial programs of the schools of all levels. LuNa didactic modules are intended to support and integrate but never to substitute classroom frontal lectures.

Further author information: (Send correspondence to M.B.: E-mail: maria.bondani@uninsubria.it) 
The experiments initially enclosed in the LuNa Project were chosen among the educational activities the Authors extensively tested over the years, for their effectiveness in acting as an incentive to students in the learning process. The activities themselves are prepared and conducted in close collaboration with the teachers of the classes involved, so as to constitute a significant connection opportunity between school teachers and the world of academic research and to give them the chance to update and to develop new ideas for innovative teaching methods.

The LuNa Project has some basic differences with respect to other similar projects devised to bring demonstrations in schools (mainly elementary schools) and often based on the idea that physical phenomena can be presented as a kind of magic and only at the end some explanation is given to the the audience..$^{2,3}$

In the neighborhood of our Department, several other activities are active that approach science dissemination from different points of view. For instance, the scientific stage shows acted by the group of Marco Giliberti, Marina Carpineti and Nicola Ludwig from Milano University ${ }^{4}$ address the problem of rising the interest from students by spectacular demonstrations in form of stories. On the other hand, since many years Paolo di Trapani exhibitions on natural phenomena in optics, which recently became also a stage show, ${ }^{5}$ have attracted a large number of visitors. All these activities are very effective in increasing the interest on physics (and optics in particular that is suitable for spectacular shows), but the question on how much students can actually learn from demonstrations is open. ${ }^{6}$ The method of LuNa Project is completely different: with our Project we want to pursue purely didactic objectives by making more attractive classroom activities and curricular programs.

The LuNa Project is organized in several independent and modular courses linked by a common thread: the nature of light, its phenomenology and its interaction with matter. Some topics lend themselves to the inclusion in most school curricula, being they obviously dealt with in more or less detail depending on school level. These paths typically unfold from the observation of phenomena peculiar to the daily experience of each student (e.g. atmospheric phenomena) to get to the presentation of the physical model explaining the phenomenon, whose level of mathematical formalization varies depending on the mathematical skills of addressed students.

Some modules (e.g. the paths on Holography and Quantum Physics) require a more specific preparatory knowledge, and are therefore reserved to the more mature audience of Secondary School Grade II.

Integral part of the teaching proposal is the activity for the choice of the university faculty, which is the ideal conclusion of Secondary School studies.

In the years 2009 and 2010, the Project was financed by both private and public sponsors that supported the acquisition of the experimental equipment but it has not been financed in the last two years. The Project has involved about 200 classes (more than 4000 students till now (four academic years)).

\section{DIDACTIC ROUTE}

The experience gained over the years taught us that an external didactic action leaves significant traces in the students only if it is perceived as an integral part of their education curricula, or if it is properly prepared and supported by the teachers of the class. For this reason, teachers interested in the various LuNa actions are invited to preparatory lectures during which the content of the different modules are presented with the purpose to be harmonized in the ordinary teaching program. In such a way, the necessary preliminary preparation of the students, obviously at different levels depending on the types of schools, will be mainly carried out by their own teachers, while all LuNa actions will be of experimental nature.

Within the Project we have developed and implemented several experiments and small-scale models of physical phenomena that show both wave and particle nature of light. These devices, of different structural and conceptual complexity, have been devised to be easily installed directly in schools and represent the core of each LuNa module. Therefore, an important part of the Project consists in the study and engineering of the equipment to allow the apparatuses to be compact, easy to transport, assemble and disassemble.

The complexity of the setups differs from experiment to experiment: in some cases, optical phenomena can be displayed and studied by using very simple and cheap material (see Fig. 1 for samples of the material used for geometrical optics), in other cases, more technically complex and expensive apparatuses are needed (see below the setups for interference and holography). 


\section{CONTENTS}

The debate on the nature of light has its roots in the history of science and is an interesting example of how the development of a model in the field of physics proceeds: the progressive addition of experimental evidence changes the concepts previously acquired by integrating them into a more complete theory. In particular, the corpuscular interpretation of light was overtaken in the XIX century by the wave interpretation which, however, could not explain all the experiments. In the early XX century the corpuscular model came back again and became clear that both concepts, wave and particle, are needed to explain the complexity of the phenomenon of light (wave-particle duality). The approach of some of the LuNa modules is supported by historical considerations to help students understand the scientific method.

In the following Subsections we list the different LuNa modules.

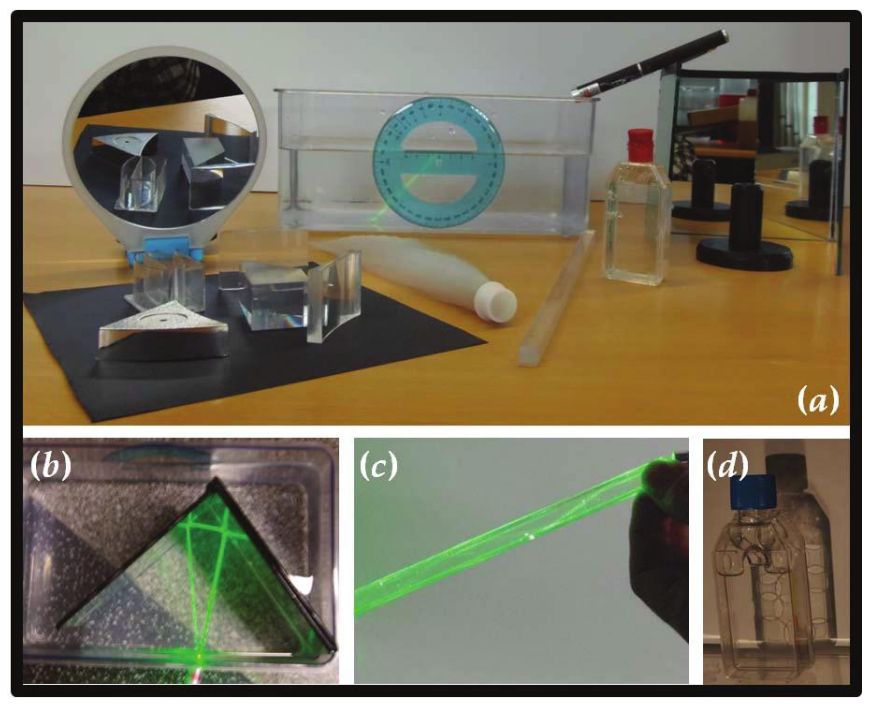

Figure 1. (a) Simple material to investigate geometrical optics; (b) light rays build up the orthic triangle; $(c)$ demonstration of a waveguide; $(d)$ transillumination to view transparent objects.

\subsection{Primary School}

\section{E1. Light in natural phenomena}

The module is focused on some luminous phenomena that are part of our everyday experience and that are originated by the interaction of light with materials, such as the color of objects, sun, sky, air and shadows (see Fig. 2). Natural phenomena will be reproduced "indoor" by realizing small-scale models of them. ${ }^{7}$

The physical description of the phenomena is addressed by introducing at a basic level the concepts of light beams and waves as interpretative models of the nature of light, wavelength and color, reflection, refraction and dispersion.

\section{E2. Light and human eye}

The module describes the operation of the eye up to the formation of images on the retina, also introducing image formation defects (myopia, hyperopia and presbyopia and astigmatism) and their correction by means of lenses. It also describes the function of the brain in visual perception and, in particular, it discusses the perception of three-dimensionality through binocular vision. The difference between photographs and holograms (that are shown to kids) is also presented. 


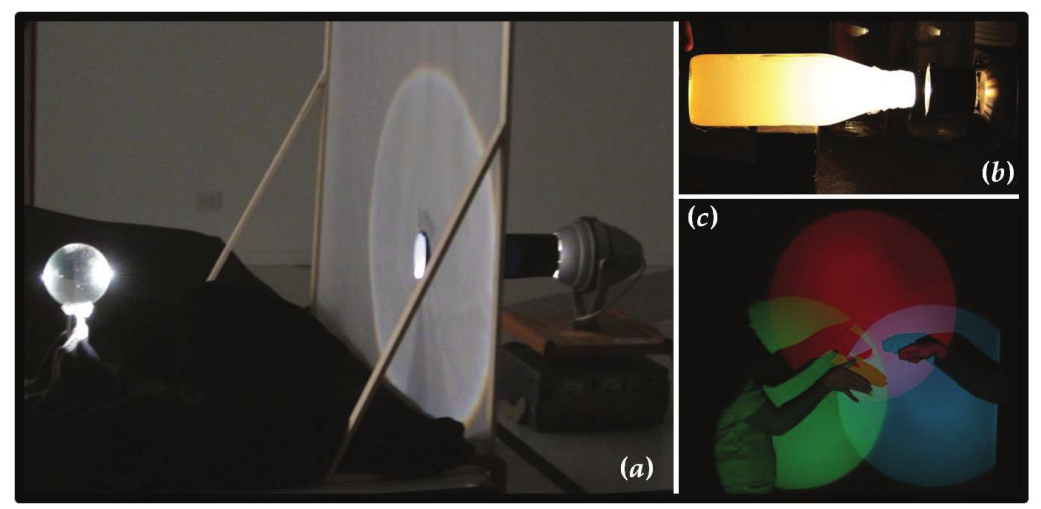

Figure 2. Indoor reconstruction of natural phenomena: (a) rainbow; (b) sunset in a bottle; (c) colored shadows.

\subsection{Lower Secondary School (11-14 year old students)}

\section{M1. Fundamentals of geometrical optics and wave optics}

The module sets out in more extensive way the themes proposed for the Primary School (E1). The goal is to reproduce in the laboratory some fundamental phenomena of geometrical and wave optics with particular attention to the corpuscular (reflection and refraction) and wave (interference and diffraction) interpretation of light, and natural phenomena arising from the interaction of light with matter (rainbow color of the sky). To help understand the peculiar behavior of waves, we use to observe mechanical waves in water with a ripple tank.

\section{M2. Vision and image formation}

The module deals with the description of the mechanism of human vision as in E2, but introducing a more quantitative description (geometrical and analytical) of image formation, based on the physical phenomena of refraction and reflection, that is very complex and involves not only the eye but also the perception of images by the brain. Regarding the analysis of the mechanism of vision, the psychological aspects related to perception are introduced, showing how perception can be cheated by using combinations of colors and shapes (optical illusions).

\section{M3. Light-matter interaction}

The aim of the module is to provide some laboratory experiments on light/matter interaction in order to justify the existence of atomic energy levels and molecular energy bands. To this purpose students will observe with simple spectroscopes the light emitted by different light sources: discharge lamps (lines), fluorescent lamps (bands), incandescent light bulbs and sun (continuous). The physical interpretation of the phenomena requires a simple discussion of atomic and molecular models, absorption, emission (fluorescence and phosphorescence) and spectra.

\subsection{Upper Secondary School (14-19 year old students)}

\section{S1. Fundamentals of geometrical and wave optics}

The module incorporates the themes proposed for the Primary School (E1) and that for the Secondary School Grade I (M1) to provide an introduction to the fundamental phenomena of geometrical and wave optics, with particular attention to the corpuscular (reflection and refraction) and wave (interference and diffraction) description of light, and to natural phenomena arising from the interaction of light with matter (rainbow color of the sky). The course is presented by simplifying the mathematical aspects so as to make it accessible to all Secondary Schools, including those non-scientifically oriented.

An extension of this module (S1b) is devoted to students of scientifically oriented schools who usually have a broader mathematical and physical background. The module deals with the same topics as S1, giving more space to the quantitative verification of the physical laws that govern the presented phenomena (quantitative verification of Snell law, calculation of the limit angle for the rainbow, calculation of interference patterns). Because 
some of the proposed experiments are easy to replicate with materials normally available to the laboratories of many high schools, the LuNa action can serve as a incentive for further studies directly managed by teachers.

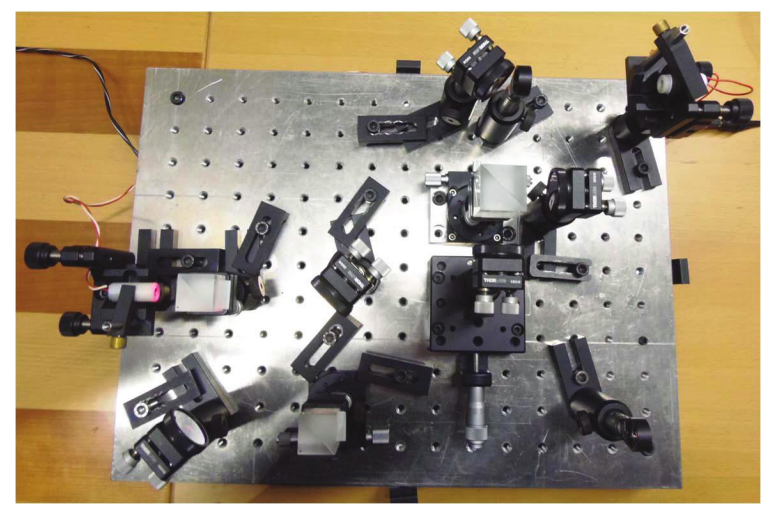

Figure 3. Portable setup including a Mach-Zehnder and a Michelson interferometer.

\section{S2. Vision and image formation}

The topics are the same as M2, but with the extension to systems of more lenses. In particular, the realization of a telescope and a microscope is proposed. Concepts of wave optics (diffraction) will be also retrieved to introduce the idea of spatial filtering of a light beam due to the presence of openings (such as the aperture of the cameras). ${ }^{8}$ An extension of this module ( $\mathrm{S} 2 \mathrm{~b}$ ) includes more detailed discussion of the perception of images by the brain, the psychological aspects related to perception and optical illusions.

\section{S3. Color theory}

The module presents the mechanism of vision related to the perception of colors. In this module we present and discuss the measurable quantities by which it is possible to describe colors and the colorimetric systems. In particular, we present spectral decomposition of light, additive and subtractive color generation, tristimulus theory and chromaticity diagram, hue, saturation, and brightness.

\section{S4. Light-matter interaction - 1}

The topics of the module are the same as M3. The aim of the module is to support experimental evidence with the explanation of atomic structure that is introduced through the study of inorganic chemistry. More insight is related to quantitative measurements of the absorption coefficient and of the absorption spectrum. Measurements of the fluorescence decay times will be carried out at the laboratories of the Department of Science and High Technology of the University of Insubria (Como) as they require a non-portable equipment.

\section{S5. Light-matter interaction - 2}

The module deals with elastic interaction of light with matter (scattering) which describes the phenomenon of diffusion. We give a description of the intensity distribution of scattered light and analyze its dependence on the nature of the scattering medium. The description of the scattering process can be given on the basis of wave or particle theory of light and must take into account the size of the diffusing particles (Rayleigh theory of blue sky, formation of speckles, multiple scattering in the mist).

\section{S6. Non-liner optical phenomena}

The non-linear behavior of physical systems is evident in a certain range of the parameters that governs their evolution. In the case of the interaction of light with matter the fundamental parameter is light intensity. The objective of this module is the demonstration of the effects of some non-linear interactions in molecules in solution and in crystals. The activity requires the use of intense laser sources that are not portable: the classes involved in the activity have to come to the Department of Science and High Technology of Insubria University (Como) where the light sources are available. 
In this module we discuss two-photon absorption of molecules in solution, saturable absorbers, parametric phenomena in nonlinear crystals (sum and difference frequency generation, parametric down-conversion (see Fig. 4)).

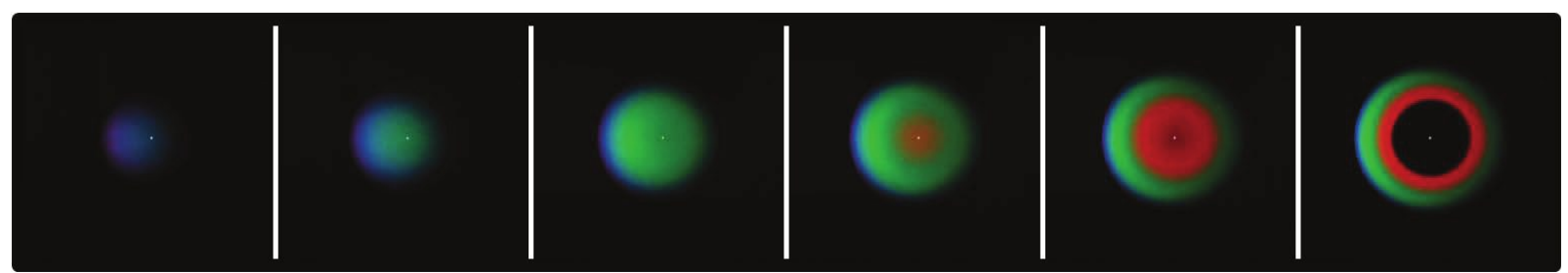

Figure 4. So-called "down-conversion cones" generated by a BBO nonlinear crystal pumped with a pulsed UV laser beam. The sequence of images is obtained by rotating the crystal with respect to the direction of the pump beam.

\section{S7. Holography}

The words "holography" and "holograms" are commonly known and stimulate students' imagination, but rarely we encounter people who have a realistic idea of what they mean: this generates misunderstandings and false expectations. For this reason, the topic of holography is somehow paradigmatic as it lends itself to a discussion of the proper methodological approach to physics that can be extended to other physical contexts. The aim of the module is the realization of holograms of small objects by using a continuous-wave laser, the photographic processing of the holographic plate and its reconstruction. We realized a portable setup (see Fig. 5), which does not require an extreme stability, so as to implement the entire process of hologram production directly in the schools. ${ }^{10}$

Some extensive theoretical introduction is required that is included in the LuNa action: definition of the hologram as an interference pattern and formal derivation of the presence and nature of the images reconstructed by the hologram; description of the photosensitive material and of the registration process; chemical processing of the holographic plates; description of the procedures for reconstructing the holographic image (real or virtual).

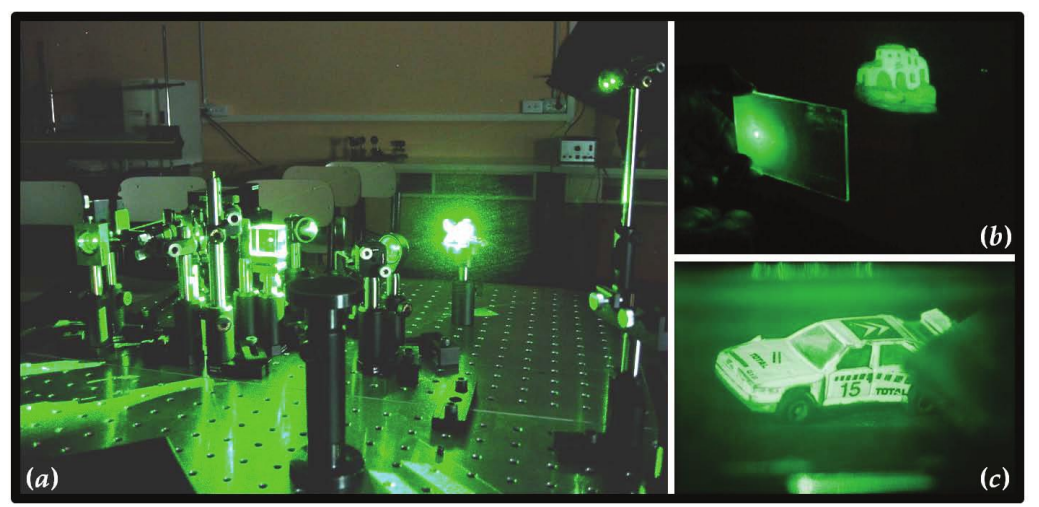

Figure 5. (a) Portable setup for registration of transmission holograms; $(b)$ reconstruction of the real image of a transmission hologram; $(c)$ reconstruction of the virtual image of a transmission hologram.

\section{S8. Demonstration of the optical wave-particle duality}

The idea of the module is to create a teaching path on modern physics based on the properties of light, to show the effects of the superposition principle and of the wave-particle duality. The contents of the module are partially non-conventional and usually ignored by High-School textbooks, For this reason some preliminary introductory work with teachers is needed (two meetings of 2 hours) to ensure that the LuNa action is properly incorporated into the curriculum. The theoretical introduction, which is integral part of the module, includes: brief historical presentation of the crisis of classical physics and the fundamental characteristics of the quantum 
description of reality; introduction to the nature of light and to the logic of the experiment that follows the reasoning described by Prof. G.C. Ghirardi in the book "Sneaking a Look at God's Cards". ${ }^{11}$

The experiment consists in the direct measurement of the interference pattern at the single-photon level by using polarized light and a Mach-Zehnder interferometer incorporating polarizing beam splitters. ${ }^{12}$ The experiment is absolutely similar to Young double-slit interference.

Also in this case we realized a portable setup to bring the LuNa action to the schools. ${ }^{12}$

\section{S9. Historical experiments of modern physics}

Aim of the module is the reproduction of some experiments that are at the basis of modern physics. We are mainly interested in highlighting the corpuscular properties of radiation and the wave properties of matter. The equipment required to perform the experiments is transportable and made available by the Department of Science and High Technology. Each interested school may request the execution of one or more experiments, taking into account that each of them needs to be properly introduced. In particular we present: measurement of black body spectrum, measurement of photoelectric effect, observation of atomic spectra, diffraction of electrons in comparison with light diffraction (see Fig. 6).

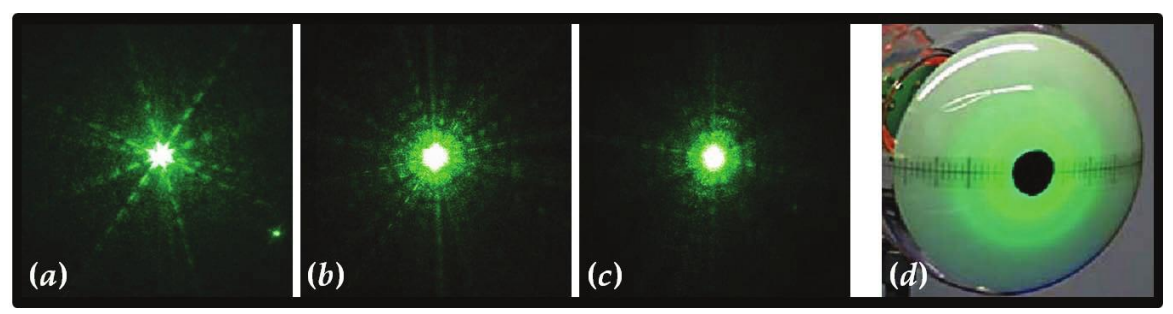

Figure 6. Electron diffraction by amorphous graphite layers is similar to light diffraction from several layers of tulle. Light diffraction by tulle: $(a)$ one layer, $(b)$ three layers, $(c)$ five layers; $(d)$ electron diffraction by amorphous graphite.

\section{RESULTS}

The Project was proposed for the first time in 2009 to the schools in the neighborhood of Como (Northern Italy) and has involved 200 classes (more than 4000 students) till now: 16 classes were from Primary Schools, 28 from Secondary Schools I grade and 156 from Secondary Schools II grade. The fact that the large majority of LuNa actions is being required by Secondary Schools, mostly scientific oriented, is not surprising because physics is often perceived as to difficult by the largest part of non specialized teachers, who not even imagine they can propose physical topics to their students. Several schools participated in the Project for subsequent years, thus testifying the appreciation of the LuNa activities.

As to the modules, S1 and S1b are by far the most required, either as an introduction of a new topic during curricular lectures or as a summary at the end of the lectures to clarify and expand the program. We notice that, in spite of the difficulty of the topic, holography is also greatly appreciated and asked due to the fascination holograms always arise.

Other modules are required more rarely and within non-curricular activities to increase and deepen the knowledge on a particular topic by exploiting the curiosity of the students. This happens in very specific contexts in which the curricular program has been completed by teachers.

During the years, some spin-off activities were triggered by LuNa Project, such as monographic courses on rainbow $^{7}$ and on photography and holography,${ }^{8,10}$ which became self-standing activities for off-hour and summer activities.

According to some informal investigations of teachers, students participating in LuNa activities are always rather satisfied by them and result to be more interested in optical phenomena. This is testified by the fact that several students decided to deepen their knowledge on some aspects of the presented topics and produced some papers to present for the diploma examination. Once again the most appreciated topics were holography and singlephoton interference, as they also lend themselves to inter-disciplinary considerations. Moreover, entire classes of younger students (Lower Secondary School) were involved by their teachers in the preparation of the activities of Open Days and School exhibitions in which they reproduced some experiments of the LuNa Project, typically atmospheric phenomena, colors and shadows. 


\section{CONCLUSIONS}

After four-year activities, we can conclude that strong points of the Project are the scientific and didactic level of the modules and their versatility that allow their integration in the different curricula. On the other hand, the aspect of LuNa modules most appreciated by both students and teachers, is the opportunity they give to experience physical phenomena directly.

Among the weak points of the Project as it has been carried out till now, we mention the difficulty in establishing a full collaboration with teachers in the preparation and integration of LuNa actions in the regular curricula. Frequently teachers tend to use the Project activities as a substitute of the experimental part of the didactic work and do not even want to get involved in the preparation and elaboration of the activity, nor in some follow-up activity. As a result, we have very few feedbacks concerning the didactic outputs of LuNa modules. Moreover, one of the original aims of LuNa activities was to try to modify the standard teachers' approach to teaching physics, which mainly employs frontal lectures, by supporting them in the introduction of some experimental activity in their lessons. Unfortunately very few teachers tried to replicate LuNa experiments. Nevertheless, from time to time the collaborations with teachers have been very fruitful, mainly in the Lower Secondary Schools.

We can thus conclude that the activities of LuNa Project seem to be effective to support conventional teaching as they give the students the opportunity to directly experience some physical phenomena. On the other hand, even if the LuNa communication style could be in principle replicated by teachers, this happens very rarely, probably due to the lack of familiarity of teachers with experimental activities. For this reason, we judge it necessary to spend more time and energies in the training of the teachers involved in the Project.

\section{ACKNOWLEDGMENTS}

The Authors acknowledge the support of Fondazione Banca del Monte di Lombardia (Pavia), the Rector of the Università degli Studi dell'Insubria and the Banca di Credito Cooperativo di Cantù financing the start-up of the Project in the period 2009-2010.

\section{REFERENCES}

[1] Website of the Project: http://luna.dfm.uninsubria.it/

[2] Curticapean, D., "University for Children - The Magic of Light," Proceedings of The Education and Training in Optics and Photonics Conference (ETOP) - Eleventh International Topical Meeting on Education \& Training in Optics and Photonics, St. Asaph, North Wales, United Kingdom, 5-7 June 2009, 1.4.66 (2009).

[3] Donnelly, J. and Magnani, N., "Outreach Magic Workshop," SPIE Optics and Photonics, 2012 - Workshops for Optics Educators - San Diego (CA, USA) - 12-16/8/2012. http://www.optics4kids.org/osa.o4k/media/optics4kids/SPIE-2012-Outreach-Magic-doc.pdf.

[4] Carpineti, M., Cavinato, M., Giliberti, M., Ludwig, N. and Perini, L., "Theatre to motivate the study of physics," Jcom 10(1), A01 (2011).

[5] http://www.diluceinluce.eu/index.php

[6] Crouch, C. H., Fagen, A. P., Callan, J. P. and Mazur, E., "Classroom demonstrations: Learning tools or entertainment?," Am. J. Phys. 72(6), 835-838 (2004).

[7] Bondani, M. and Favale, F., "Dismantling rainbow," Proceedings WCPE2012 (2013). In press.

[8] Bondani, M. and Favale, F., "Following the path of light: recovering and manipulating the information about an object," this SPIE Proceedings Issue.

[9] Allevi, A., Bondani, M., Ferraro, A. and Paris, M.G.A., "Classical quantum aspects of multimode parametric interactions," Laser Physics, 16(10), 1451-1477 (2006).

[10] Favale, F. and Bondani, M., "School outreach program for teaching optics: let's start with holography," Proceedings WCPE2012 (2013). In press.

[11] Ghirardi, G. C., "Sneaking a Look at God's Cards, Revised Edition: Unraveling the Mysteries of Quantum Mechanics," (translated by Gerald Malsbary, Princeton University Press, Princeton, New Jersey USA, 2007). Original edition in italian: G. C. Ghirardi, "Un'occhiata alle carte di Dio" (Il Saggiatore, Milano, Italy, 2001).

[12] Bondani, M., "Single-photon interference experiment for High Schools," this SPIE Proceedings Issue. 